\title{
Photosynthetic Electron Transfer by Dint of Protein Mobile Carriers. Multi-particle Brownian and Molecular Modeling
}

\author{
Galina Riznichenko, ${ }^{1,}$, Ilya Kovalenko ${ }^{1}$, Vladimir Fedorov ${ }^{1}$, Sergei Khruschev $^{1}$, Andrey Rubin ${ }^{1}$ \\ ${ }^{1}$ Dept Biophysics, Biological Faculty, Lomonosov Moscow State University, RU-119234 Moscow, Russia
}

\begin{abstract}
The paper presents the review of works on modeling the interaction of photosynthetic proteins using the multiparticle Brownian dynamics method developed at the Department of Biophysics, Biological Faculty, Lomonosov Moscow State University. The method describes the displacement of individual macromolecules - mobile electron carriers, and their electrostatic interactions between each other and with pigment-protein complexes embedded in photosynthetic membrane. Three-dimensional models of the protein molecules were constructed on the basis of the data from the Protein Data Bank. We applied the Brownian methods coupled to molecular dynamic simulations to reveal the role of electrostatic interactions and conformational motions in the transfer of an electron from the cytochrome complex (Cyt $b_{6} f$ ) to the molecule of the mobile carrier plastocyanin (Pc) in plants, green algae and cyanic bacteria. Taking into account the interior of photosynthetic membrane we developed the model which combines events of proteins Pc diffusion along the thylakoid membrane, electrostatic interactions of Pc with the membrane charges, formation of Pc super-complexes with multienzyme complexes of Photosystem I and Cyt $b_{6} f$, embedded in photosynthetic membrane, electron transfer and complex dissociation. Multiparticle Brownian simulation method can be used to consider the processes of protein interactions in subcellular systems in order to clarify the role of individual stages and the biophysical mechanisms of these processes.
\end{abstract}

\section{Introduction}

Photosynthetic electron transport forms the basis of light energy transduction into energy of chemical compounds, ATP and NADPH, used for metabolic needs, firstly, for $\mathrm{CO}_{2}$ assimilation in the chloroplast of leaves and algae. Methods of mathematical and computer modeling significantly contribute to the research of the mechanisms underlying the different steps of photosynthetic electron flow to enrich the knowledge on the physic-chemical basis of energy transduction.

Traditional kinetic models, aimed at the description of the electron transport chain use the apparatus of ordinary differential equations based on master equations and the mass action law (review see [1-4]). However, the structure of the photosynthetic membrane (Fig 1) shows that in areas where electron transport is carried out by mobile carriers (molecules of the PQ pool, Pc in the lumen, Fd on the stromal side of the PSI) their interaction with multi-enzyme complexes does not correspond to the concepts of free diffusion and random collisions. Thus, in a narrow luminal space, the Pc molecules, whose dimensions are comparable to the width of the luminal space, can't move freely [5].

To simulate the interactions of mobile electron carriers with multi-enzyme complexes in photosynthetic membrane the method of 'direct multi-particle modeling' was developed at the Lomonosov Moscow State University by the Department of Biophysics of the
Biological Faculty together with the Department of Computer Methods in Physics of the Physics Faculty. The main idea of this approach and the results obtained are presented in the papers [4, 6-12].

The method describes the displacement of individual macromolecules - mobile electron carriers, and their interaction between each other and with pigment-protein complexes embedded in photosynthetic membrane. Three-dimensional models of the protein molecules were constructed based on the data from the Protein Data Bank. To simulate translational and rotational motion of molecules under the influence of random and electrostatic forces in a viscous medium the Langevin equations were used as described in [7]. To determine the electrostatic potential grid around each molecule Poisson-Boltzmann calculations [13] were used as described in [14]. For simulation of multiparticle BD original software ProKSim [15, 16] was used. The program is available by request.

For the comparison with experimental data, such as the absorption spectrum of cell suspension, it is necessary to consider ensembles of individual macromolecules. Molecules of electron carrier proteins perform Brownian motion in a medium and undergo electrostatic interactions with each other and with the surface of a photosynthetic membrane. When the donor and acceptor molecules approach each other, they are able to form a transient oxidation-reduction complex.

e-mail: riznich46@mail.ru

(C) The Authors, published by EDP Sciences. This is an open access article distributed under the terms of the Creative Commons Attribution License 4.0 


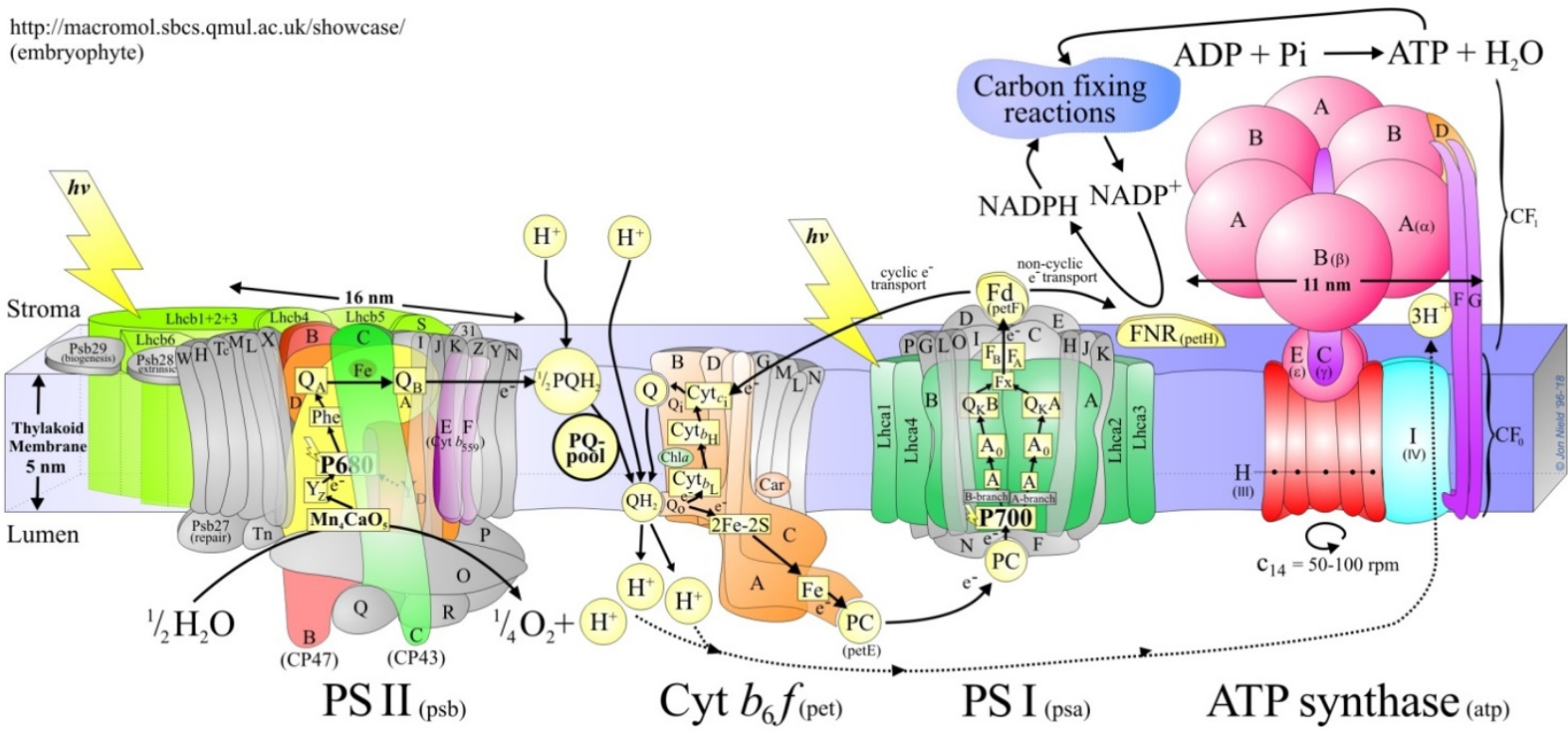

Fig.1. Multienzyme complexes in photosynthetic membrane. The arrows show the paths of electron transfer. (Jon Nield, http://macromol.sbcs.qmul.ac.uk/showcase/)

As a result of conformational changes, the final complex is created and an electronic path is formed, which ensures electron tunneling from the donor molecule to the acceptor molecule. To describe conformational motions in such a reaction complex, it is necessary to use methods of molecular dynamics, and methods of quantum chemistry to describe an electron transfer from the reaction center of a donor molecule to the reaction center of an acceptor molecule.

Models of Brownian dynamics (see review [12]) simulate the diffusion and mutual orientation of protein molecules in the formation of a preliminary complex. The distinctive feature of our models is that they are multi-particulate and allow us to simulate not only the processes of interaction of proteins in solution, but also to study the behavior of ensembles of interacting macromolecules in the complex interior of the photosynthetic membrane and to take into account the structural features of the membrane organization $[14,16$, 17].

Along with kinetic characteristics that can be obtained on the traditional kinetic model the direct model provides a three-dimensional visual representation of the processes in the system at different spatial and temporal scales. It makes it possible to observe the behavior of the individual components and obtain statistical information about the system. For example, we calculate every time moment, number of certain molecules that are oxidized or reduced, a number of formed complexes and the average energy of the electrostatic interactions of the molecules of that complexes.

With the help of the multi-particle Brownian dynamics, the interaction of the Pc molecule with the cytochrome $\mathrm{f}$ cofactor in a solution $[7,18]$ and in the lumen of the thylakoid $[14,16]$, the interaction of Pc with the PSI donor part and the interaction of Fd with the
PSI acceptor part [9] and Fd with subsequent electron acceptors, FNR [8], and hydrogenase [19-21]) were simulated (Fig. 2.)

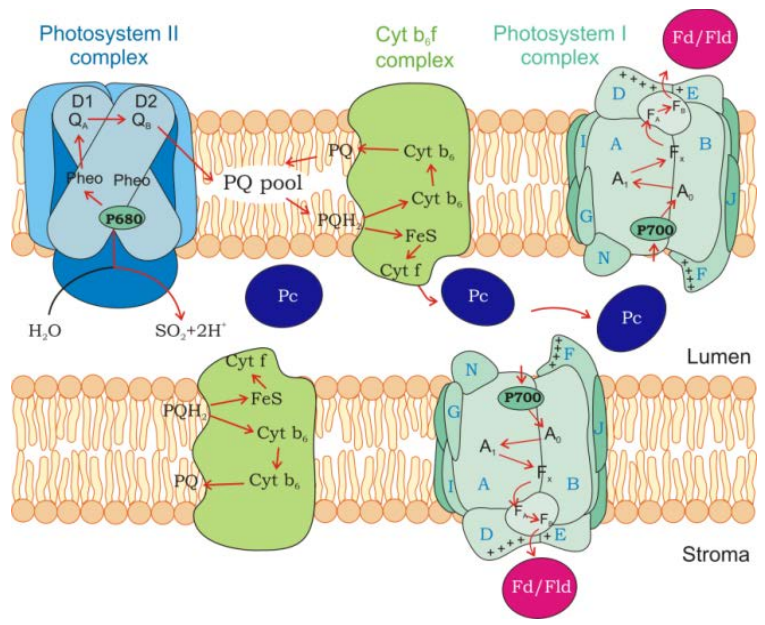

Fig. 2. Schematic view of photosynthetic electron transport processes. Two thylakoid membranes and the luminal space between them are shown. Multi-protein complexes Photosystem 1, Photosystem 2, and cytochrome bf are embedded in the membrane. Molecules of mobile electron carrier protein plastocyanin (Pc) diffuse in the lumen. The arrows denote electron transport. The connection with the Calvin cycle is carried out by mobile carriers Fd (or Fld).

\section{Stages of protein-protein interaction}

The electron transfer between proteins involves several stages (Fig. 3): 


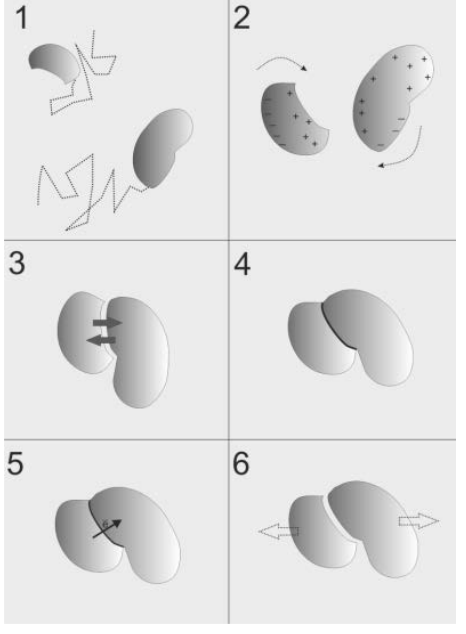

Fig.3. Stages of electron transfer between proteins in solution. 1. Diffusion motion (translational and rotational). 2. Mutual orientation of interacting proteins under the action of Brownian and electrostatic forces. 3. Formation of a mutual configuration ('preliminary complex'). 4. Transition from the preliminary to the final complex. 5. Transfer of an electron between reaction centers of donor and acceptor proteins. 6. Decay of the complex and formation of a pair of proteins

(1) The diffusion motion (translational and rotational) of a protein under the influence of a random Brownian force to the docking site or the movement of a moving protein to another protein that is a part of the multienzyme complex embedded into the membrane. While proteins are at the relatively large distances from each other, electrostatic interactions between them can be neglected, since electrostatic forces are very weak due to screening of the field by water molecules and ions. In our models, we did not take into account electrostatic interactions at the distances greater than $35 \AA$ [22].

(2) Mutual orientation of interacting proteins under the action of Brownian and electrostatic forces. At distances less than $35 \AA$, the electrostatic forces due to interaction of protein surface charges become comparable with the Brownian force. This distance is called the distance of the electrostatic interaction. At shorter distances convergence of proteins (possibly multiple) leads to their mutual orientation. Here an important role is played by rotational motion.

(3) When the reaction centers of two proteins converge for a distance called the 'interaction radius', a mutual configuration 'preliminary complex' is possible, which can then turn into the 'final complex' where the electron transfer reaction takes place. The preliminary complex can also split and then individual proteins continue Brownian motion.

(4) The transition from the preliminary to the final complex is due to the inclusion of van der Waals interactions and conformational motions of the components of the preliminary complex at these distances.

(5) In the final complex, electron tunneling between reaction centers of the donor and acceptor proteins becomes possible.

(6) After carrying out the electron transfer reaction, the complex again undergoes conformational transitions leading to the splitting of the complex thus forming a pair of proteins, where the former donor is oxidized and the former acceptor is reduced. Protein molecules are ready to participate in the next act of electron transfer. The Brownian/molecular models [23] have described stages 14 of the process. The formation of the final reaction complex is preceded by a sequence of processes that ensure the conformational correspondence of donor and acceptor molecules. To provide conditions for electron tunneling from a donor molecule to an acceptor molecule, interacting proteins must properly orient themselves mutually, as to 'get properly attached' to each other. The experimentally observed and simulated dependence of the reaction rate on ionic strength indicates the important role of electrostatic interactions in the formation of encounter complex [14, 24]

\section{Productive and encounter complexes}

non-productive

To reveal in detail the mechanisms of encounter complex formation, the classical approach of Brownian dynamics was used, namely multiple computational experiments on the convergence of two molecules. The protein movement was simulated by the software ProKSim - Protein Kinetic Simulator [15]. The analysis of molecular trajectories in such experiments makes it possible to identify highly frequent types of their mutual arrangement that form most often during the approach of diffusive molecules to estimate the interaction energy between molecules and the rate constant of the protein association reaction.

The role of electrostatic interactions in the formation of the oxidation-reduction complex of two proteins was studied in the interaction model of the well-studied pair of photosynthetic electron transfer proteins, Pc and Cyt $f$. This pair of proteins is a classic object of experimental research (reviewed by Cruz-Gallardo et al. [25], and Khruschev et al. [12]) and Brownian dynamics studies [13, 18, 23, 24, 26-29]. The physiological function of the protein Pc consists of the shuttle electron transfer between the subunit $\mathrm{f}$ of cytochrome complex b6- $\mathrm{f}$ and the photosystem I in all higher plants and some algae. Cytochrome $f$ of the cyt $b_{6} f$-complex contains the Pc binding site. Proteins Pc and cyt $f$ are oxidationreduction partners having well-localized reaction centers - copper and iron atoms, respectively. To analyze the mutual distribution of macromolecules in the ensemble of final configurations obtained by Brownian dynamics, factor and cluster analysis methods were used [24]. An analysis of several thousand structures showed that during the diffusion process two types of encounter complexes are formed (Fig. 4).

In the cluster of 'correct' configurations (Fig. 4, at the center), the mutual orientation of the molecules Pc and Cyt $f$ corresponds to the orientation in the functionally active complex, determined experimentally (Fig. 4, left, PDB ID: 2PCF structure, NMR data [26]). In complexes of this cluster Pc atoms have sufficient mobility, to carry out the further approach of active centers of the molecules. In the cluster of 'irregular' 
configurations (right), the mutual orientation of the molecules Pc and Cyt $f$ does not correspond to the orientation in the functionally active complex, and the Pc atoms have very limited mobility.

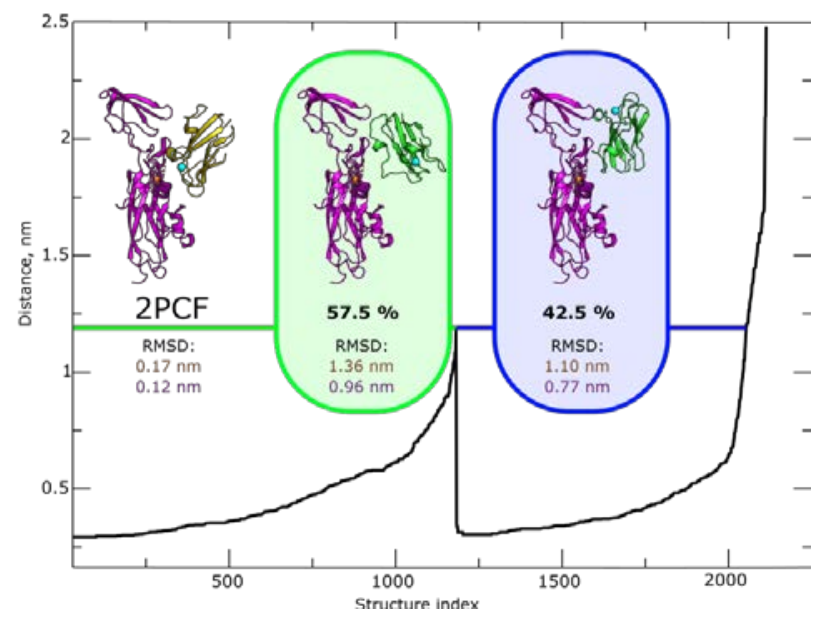

Fig. 4. Structures of encounter protein complexes of plastocyanin and cytochrome $f$ with electrostatic attraction energy more than $8 \mathrm{kT}$. Left - experimentally determined structure from PDB. Centre - 'productive' simulated configuration. Right - 'unproductive' simulated configuration. The plot of the reachability distance represents the hierarchical tree with two well separated branches obtained by the densitybased clustering [24].

\section{Brownian I Molecular Models of Photosynthetic Protein Interaction}

For electron transfer, it is necessary that in the process of conformational changes from the diffusion-collision complex a final complex is formed, in which the reaction centers are at a distance close enough for the electron tunneling from the donor molecule reaction center to the acceptor molecule. Molecular dynamics provides the means for modeling the conformational movements of molecules.

Sequential processes of diffusion convergence of proteins, their mutual orientation and further conformational 'adjustment' leading to the formation of the final complex were modeled in two stages. First, with the Brownian dynamics method we repeatedly simulated the processes of approach and mutual orientation of molecules. The cluster analysis of a large ensemble of tens of thousands of trajectories of the Brownian motion revealed various energetically favorable metastable states in the process of the formation of a complex of proteins of the electron donor and electron acceptor. Then, the final configurations of molecules for the most frequently encountered trajectories were used as initial configurations for molecular dynamics. At this second stage the formation of the final complex occurred (or did not occur) in which tunneling electron transfer is possible.

Computer simulation of the complex formation of photosynthetic proteins Pc and Cyt $f$ was carried out for different types of organisms: higher plants, green microalgae and cyanobacteria. Cluster analysis revealed metastable conformations, which were compared with structures obtained experimentally by NMR. The structures of the Pc-Cyt- $f$ complexes for green plants, green algae and two cyanic species shown in the Protein Data Bank are shown in Fig. 5.

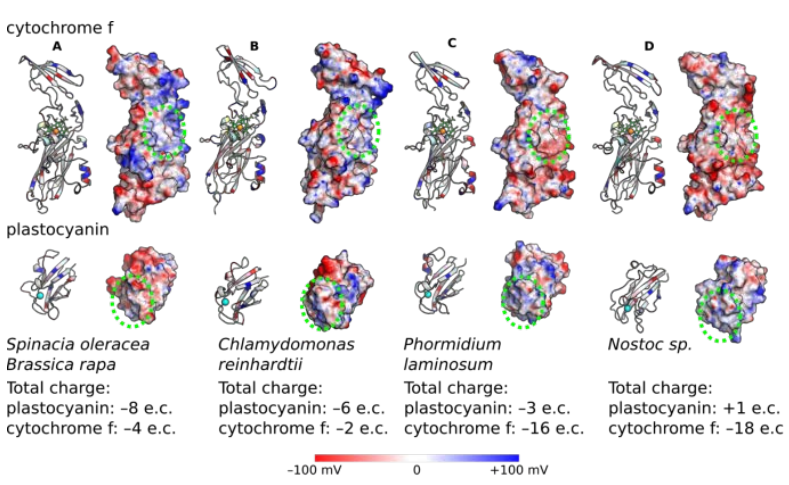

Fig. 5. Secondary structures and equipotential surfaces of proteins. Surfaces are colored according to the electric potential field from $-100 \mathrm{mV}$ (red) to $+100 \mathrm{mV}$ (blue). Contact areas are marked in green. The protein structures are oriented so that contact zones are visible. Secondary structures are colored by the type of amino acid residues of proteins. Hydrophobic residues are colored gray, aromatic pink, polar light blue, positive blue, negative red, cysteine yellow, proline green. The spheres are atoms of iron (orange) and copper (cyan) [23].

Fig. 5 shows that proteins of higher plants and green algae have different charge distribution in the contact area compared with proteins from cyanobacteria. Complexes of cyanobacteria are very different from each other in their total charge on proteins. However, in all these organisms the electron transfer from the protein Cyt $f$ to the protein Pc occures.

Brownian and subsequent MD simulation [23] showed that the role of electrostatic interactions and conformational changes in the formation of complexes of Pc and Cyt $f$ proteins is different in organisms with various forms of the photosynthetic apparatus. In higher plants and green algae, electrostatic interactions contribute to the occupation of a position near the cytochrome $f$ heme by the plastocyanin molecule. Further convergence of protein cofactors and the formation of the final complex occur as a result of rotational movements of the plastocyanin molecule entrapped in the 'binding' site.

In cyanobacterium $P$. laminosum, the complex is formed in a 'collisional' type without prior orientation of the molecules. In the case of cyanobacterium Nostoc, the plastocyanin already in the course of the Brownian movement approaches the cytochrome $f$ molecule due to the long-range electrostatic interactions in the orientation in which the copper atom is facing the cytochrome $f$. We note that the rate constants of the interaction reaction $\mathrm{Cyt}$ $f-$ Pc estimated in experiments using spectral methods are significantly lower in cyanic than in higher plants. Discussion of this fact, see ([14] and Refs herein). 


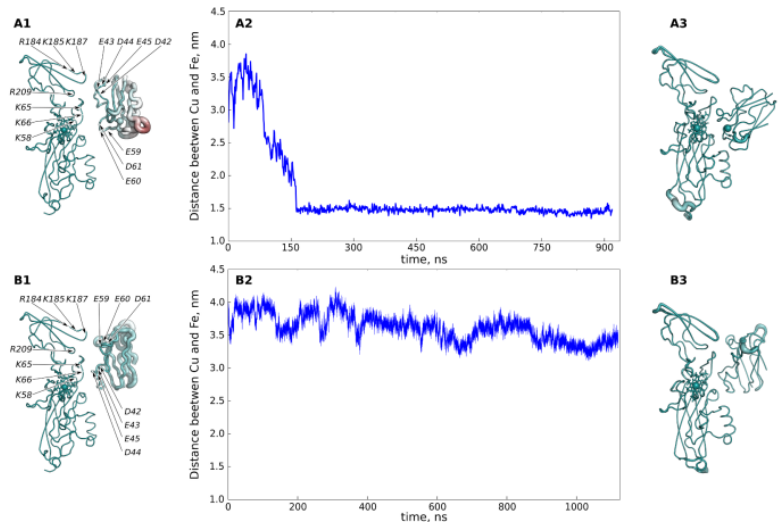

Fig.6. A1, B1: central structures of 'productive' (A1) and 'unproductive' (B1) clusters of diffusion-collisional (encounter) complexes of plastocyanin and cytochrome $\mathrm{f}$ from higher plants with an electrostatic energy of more than $8 \mathrm{kT}$. A2, B2: distance between copper and iron atoms of Pc and cyt $f$, obtained from MD calculations that have the productive (A2) and unproductive (B2) clusters as the initial structure. A3, B3: structures of the productive (A3) and unproductive (B3) final complexes obtained from MD calculations. Structures are painted by the value of the B-factor from emerald (0) to ruby (7263 $\AA^{2}$ in A1, B1 and $2074 \AA^{2}$ in A3, B3). The thickness of the lines of protein structures is proportional to the value of the B-factor [23]

In the case of green plants and green microalgae, an ensemble of structures with an energy of $8 \mathrm{kT}$ formed in the process of diffusional movement is divided into two significantly different clusters (Fig. 4). In the first cluster (Fig. 6, A) protein molecules have an orientation close to the experimentally obtained functionally active complex with an electrostatic bond between oppositely charged regions of two proteins (Fig. 5). In this case, the region of the plastocyanin molecule, which forms an electrostatic bond with cytochrome f, has a low mobility with respect to cytochrome $\mathrm{f}$, while the opposite side of the plastocyanin molecule is subject to fluctuations of greater amplitude. This is confirmed by the results of the calculation of the Debye-Waller temperature factor (Bfactor), shown in Figure 6, A1: emerald color shows areas with the lowest mobility, ruby color means maximum mobility. Thus, in the first cluster, the Pc molecule, due to rotation under the action of thermal fluctuations, has the opportunity to take a favorable position relative to cyt $f$ heme, which promotes electron transfer.

The central structure of the second cluster (Fig. 6, B) in the course of molecular dynamics leads to a rather stable, but inverted position relative to the orientation it has in the complexes obtained by NMR. The structures of the second cluster are unproductive metastable states that cannot be easily destroyed by the action of random Brownian force. Formation of such 'unproductive' complexes may lead to the decrease in the rate of electron transfer between $\mathrm{Pc}$ and Cyt $\mathrm{f}$ at low ionic strengths $[14,30]$.

\section{Simulation of electron transfer from cytochrome bf to Photosystem I}

\section{performed by mobile carriers plastocyanin in lumen of photosynthetic membrane}

Linear photosynthetic electron transport involves subsequent electron transfer from water to $\mathrm{NADP}^{+}$ through the large pigment-protein complexes embedded in the thylakoid membrane, such as photosystem II (PSII), cytochrome $b f$ (Cyt bf ), and PSI, interconnected by small mobile electron carriers, PQ inside the membrane and protein Pc in lumen (Fig.1). Diffusion and formation of the binding complex is critical to ensure electron transfer between photosynthetic proteins, in particular, from Cyt $b f$ to Pc and from Pc to PSI. The binding process depends on many factors, including diffusion rate, electrostatic properties of protein and membrane surface, distribution and concentration of reaction components, $\mathrm{pH}$, etc.

Multiparticle Brownian computer model [14, 16] of electron transfer in the thylakoid lumen from Cyt bf complex to PSI was designed to study the kinetic characteristics of the process. The simulation algorithm included the following steps [16]: (1) A simulation of Brownian diffusion of Pc molecules in the lumen between two membrane layers packed with transmembrane complexes of PSI and Cyt bf ; (2) longrange electrostatic interactions between Pc and PSI or Pc and Cyt $b f$ considered at distances of $3.5 \mathrm{~nm}$ or less which steer the mobile molecules toward orientation favoring complex formation; (3) formation of an active complex and electron transfer and (4) complex dissociation.

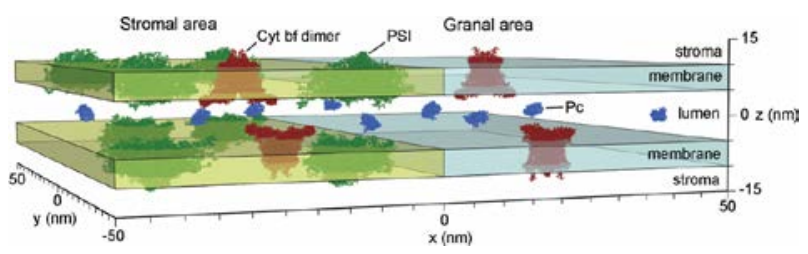

Fig. 7. Visualization of a part of the simulation area $(100 \times 100 \mathrm{~nm})$ divided into stromal region with embedded PSI and Cyt $b_{6} f$ complexes (left), and granal region with Cyt $b_{6} f$, but without PSI (right) [16]

The fragment of the scene of simulation is presented in Fig. 7. It contains two different parts: stromal with embedded PSI as well as Cyt $b_{6} f$ complexes, and granal, with embedded PSII and Cyt $b_{6} f$, but without PSI. The model predicts that Pc molecules initially located in the stroma-exposed area have the average time for docking to PSI of $19 \mu \mathrm{s}$ and for the redox reaction of $36 \mu \mathrm{s}$. It is reasonable to assume that Pc molecules in the vicinity of PSI are rapidly directed by electrostatic interactions, and the whole electron transfer process is determined mainly by the active complex formation. For the Pc molecules initially located in the granal area the average time for docking is longer by about one order of magnitude due to diffusion to the stromal part.

The experimental kinetics showed a pattern approximated fairly well by one or two exponential rise functions [5], indicating a major limitation by one or two light-induced processes. The results of simulation 
showed that the second (slow) component appeared when concentration of the reduced Pc in the vicinity of PSI becomes comparable or lower than the amount of PSI. In general, results of the simulation showed a good correlation with the experimental data.

\section{Conclusion and perspectives}

In the recent years, due to the rapid development of information technology in the modeling of various systems, the so-called 'agent' models have spread. 'Agent' (object, atomistic, corpuscular) modeling seeks to derive the properties of total complex systems from the properties and types of interactions of 'agents' - the simplest structural elements that make up this system. The 'agent' can represent a real physical object, a macromolecule that has a certain mass, volume, and charge distribution, as is the case of Brownian dynamics models. In molecular dynamics, 'agents' are atoms or their aggregates (coarse-grained modeling) [31-33] while in multiparticle Brownian modeling the 'agents' are biomacromolecules [6].

To study the biophysical mechanisms of interaction of biological macromolecules, it is necessary to simulate the processes at the subcellular and molecular levels. In this case, an important role is also played by the interior in which such interactions take place. To simulate the interaction of assemblies of macromolecules with limited mobility in a heterogeneous interior, we have developed an approach of 'direct' multiparticle modeling. We consider a three-dimensional computer stage constructed according to the contemporary data about the spatial organization of the photosynthetic membrane. The simulated molecules which move in accordance with the laws of Brownian dynamics are oriented relative to each other in electrostatic field created by the molecules themselves and by the medium. The adequacy of the model is proved by comparison of kinetic characteristics of the simulated processes with the observed experimental data.

Using data from the PDB we can 'directly' calculate the electric potential distribution around each of the interacting molecules and evaluate the role of electrostatic interactions in the process of reactiondiffusion (encounter) complex formation in the oxidation-reduction reaction. Molecular dynamics simulation allows us to follow the conformational movements in the coupled donor and acceptor molecules during the process of finite complex formation.

Due to direct multiparticle modeling approach it becomes possible to take explicitly into account shape and size of the molecules and elements of the reaction volume. Multiparticle modeling helps to understand how the real physical mechanisms of molecular interactions (diffusion, electrostatics, etc.) determine the overall dynamics of the processes in a cell.

In general, the application of kinetic modeling, Brownian dynamics, multiparticle direct approach, molecular dynamics and their combination in the study of photosynthesis appears to be promising for understanding of fundamental mechanisms of processes in living cells, and for practical purposes of the diagnosis of plant and algae cell state in biotechnology and environmental monitoring.

\section{References}

1. Photosynthesis in silico. Advances in Photosynthesis and respiration, V.29 (Springer, Dordrecht, 2009)

2. A. Stirbet, G.Yu. Riznichenko, A.B. Rubin, Govindjee, Biochemistry 79, 291-323 (2014)

3. N.E. Belyaeva, A.A. Bulychev, G.Yu. Riznichenko, A.B. Rubin, Photosynth. Res. 130, 491-515 (2016)

4. A.B. Rubin, G.Yu. Riznichenko, Mathematical biophysics (Springer, New York, 2014)

5. W. Haehnel, R. Ratajczak, H. Robenek, J. Cell Biol. 108, 1397-1405 (1989)

6. G.Yu. Riznichenko, I.B. Kovalenko, Int. Journal of Appl. Res. in Bioinformatics 9(1), 1-19 (2019)

7. I.B. Kovalenko, A.M. Abaturova, P.A. Gromov, D.M. Ustinin, E.A. Grachev, G.Yu. Riznichenko, A.B. Rubin, Phys. Biol. 3, 121-129 (2006)

8. I.B. Kovalenko, A.N. Diakonova, A.M. Abaturova, G.Yu. Riznichenko, A.B. Rubin, Phys. Biol. 7(2) (2010)

9. I.B. Kovalenko, A.M. Abaturova, A.N. Diakonova, G.Yu. Riznichenko, A.B. Rubin, Math. Model. Nat. Phenom. 6, 39-54 (2011a)

10. I.B. Kovalenko, A.N. Diakonova, G.Yu. Riznichenko, A.B. Rubin, BioSystems 103, 180-187 (2011)

11. G.Yu. Riznichenko, I.B. Kovalenko, A.M. Abaturova, A.N. Diakonova, D.M. Ustinin, E.A. Grachev, A.B. Rubin, Biophys. Rev. 2(3), 101110 (2010)

12. S.S. Khruschev, A.M. Abaturova, A.N. Diakonova, D.M. Ustinin, V.A. Fedorov, I.B. Kovalenko, G.Yu. Riznichenko, A.B. Rubin, Biophysics 60 212-231 (2015)

13. G.M. Ullmann, E.-W. Knapp, N.M. Kostic, J. Am. Chem. Soc. 119, 42-52 (1997)

14. O.S. Kniazeva, I.B. Kovalenko, A.M. Abaturova, G.Yu. Riznichenko, E.A. Grachev, A.B. Rubin, Biophysics 55(2), 221-227 (2010)

15. S.S. Khruschev, A.M. Abaturova, A.N. Diakonova, D.M. Ustinin, D.V. Zlenko, V.A. Fedorov, I.B. Kovalenko, G.Yu. Riznichenko, A.B. Rubin, Comp. Res. and Mod. 5(1), 47-54 (2013) [in Russian]

16. I.B. Kovalenko, O.S. Knyaseva, T.K. Antal, V. Ponomarev, G.Yu. Riznichenko, A.B. Rubin, Physiol. Plant. 161, 88-96 (2017)

17. D.M. Ustinin, I.B. Kovalenko, G.Yu. Riznichenko, A.B. Rubin, Comp.Res. and Mod. 5(1), 65-81 (2013) [in Russian]

18. I.B. Kovalenko, S.S. Khruschev, V.A. Fyodorov, G.Yu. Riznichenko, A.B. Rubin, Doklady RAS. Biochemistry and Biophysics 468, 183-186 (2016) 
19. A.N. Diakonova,.S.S. Khrushchev, I.B. Kovalenko, G.Yu. Riznichenko, A.B. Rubin, Phys. Biol. 13(5) 056004 (2016)

20. A.N. Diakonova, S.S. Khrushchev, I.B. Kovalenko, G.Yu. Riznichenko, A.B. Rubin, Biophysics 61, 572-579 (2016)

21. G.Yu. Riznichenko, T.Yu. Plyusnina, A.N. Diakonova, I.B. Kovalenko, S.S. Khruschev, A.B. Rubin, In: Nonlinearity: Problems, Solutions and Applications, 181-202 (U. S. Sci. Publ. Inc. 2017)

22. A. Finkelstein, O. Ptitsyn, Protein Physics (2nd Edition) (Academic Press 2016)

23. V.A. Fedorov; I.B. Kovalenko, S.S. Khruschev; D.M. Ustinin, T.K. Antal, G.Yu. Riznichenko, A.B. Rubin, Phys. Plant. 166(1), 320-335 (2019)

24. S.S. Khruschev, A.M. Abaturova, V.A. Fedorov, I.B. Kovalenko, G.Yu. Riznichenko, A.B. Rubin, Biophysics 60, 513-521 (2015)

25. I. Cruz-Gallardo, I. Díaz-Moreno, A. Díaz-Quintana, M.A. De la Rosa, FEBS Lett. 586(5), 646-652 (2012)

26. M. Ubbink, M. Ejdebeck, B.G. Karlsson, D.S. Bendall. Structure 6, 323-335 (1998)

27. D.C. Pearson Jr, E.L. Gross, Biophys. J. 75, 26982711 (1998)

28. E.L. Gross, C. Pearson, Biophys. J. 85, 2055-2068 (2003)

29. E.L. Gross, I. Rosenberg, Biophys. J. 90: 366-380 (2006)

30. T.E. Meyer, Z.G. Zhao, M.A. Cusanovich, G Tollin, Biochemistry 32, 4552-4559 (1993)

31. M. Karplus, G.A. Petsko, Nature 348, 631-639 (1990)

32. R.O. Drior, R.M. Dirks, J.P. Grossman, H. Xu., D.E. Shaw, Ann. Rev. of Biophysics 41, 429-445 (2012)

33. M. Childers, V. Daggett, Mol. Syst. Des., and Eng. 2, 933 (2017) 